\section{LEISHMANIOSES: O CONHECIMENTO DOS PROFISSIONAIS DE SAÚDE EM ÁREA ENDÊMICA}

\author{
Leishmaniasis: the healthcare professionals' knowledge in \\ endemic area
}

\author{
Leishmaniasis: el conocimiento de los profesionales de la \\ salud en area endémica
}

\section{RESUMO}

Objetivo: Investigar o conhecimento sobre as leishmanioses dos profissionais de saúde de um município endêmico do estado de Minas Gerais-Brasil. Métodos: Realizou-se um estudo transversal com 228 profissionais (95 agentes de zoonoses, 83 agentes comunitários de saúde, 18 médicos, 17 enfermeiros, 8 dentistas e 7 veterinários) do município de Divinópolis - Minas Gerais, entre julho e novembro de 2009. Utilizou-se um questionário estruturado, previamente validado, contendo questões objetivas sobre a doença. As análises foram realizadas através do programa Statistical Analysis System. Resultados: A categoria profissional que apresentou melhor média de acertos foi a dos veterinários $(8,3)$, seguida pela dos médicos $(8,1)$, enquanto os agentes comunitários de saúde tiveram a menor média $(6,7)$. As respostas adequadas com menor percentual de acerto foram: medidas preventivas (42,5\%), manifestação clínica (25,9\%), nomes populares $(20,6 \%)$ e transmissão $(20,2 \%)$. Os agentes comunitários de saúde e zoonoses, embora tenham apresentado maior porcentagem de respostas incorretas nas demais questões, foram os que mais acertaram sobre medidas preventivas, juntamente com os veterinários. Conclusão: Evidenciaram-se lacunas conceituais nos profissionais de saúde participantes da pesquisa, reforçando a necessidade de implementar processos de educação permanente destes profissionais, contextualizando as informações sobre as leishmanioses à realidade estudada.

Descritores: Leishmaniose; Conhecimento; Recursos Humanos em Saúde; Atenção Primária à Saúde; Doenças Endêmicas.

\section{ABSTRACT}

Objective: To assess the knowledge about leishmaniases among healthcare professionals of an endemic municipality of the state of Minas Gerais-Brazil. Methods: Cross-sectional study conducted with 228 professionals $(95$ zoonosis professionals, 83 community health agents, 18 doctors, 17 nurses, 8 dentists and 7 veterinarians) of the municipality of Divinópolis - Minas Gerais between July and November 2009. It was used a structured questionnaire, previously validated, containing objective questions about the disease. Analyses were performed using the Statistical Analysis System. Results: The professional category that obtained the best mean scoring answers was the veterinarians (8.3), followed by the doctors (8.1), while community health agents had the lowest mean scoring (6.7). The adequate answers with the lowest percentage of correct answers were: preventive measures (42.5\%), clinical manifestations (25.9\%), popular names (20.6\%) and transmission $(20.2 \%)$. Although zoonosis professionals and health community agents presented the highest percentage of wrong answers, they were the ones - along with veterinarians - who answered most of the questions about preventive measures right. Conclusion: Conceptual gaps were observed among the healthcare professionals participating in this research, reinforcing the need to implement continuing education processes for these professionals, contextualizing the information on leishmaniases according to the reality studied.

Descriptors: Leishmaniasis; Knowledge; Health Manpower; Primary Health Care; Endemic Diseases.
Artigo Original 1) Centro de Pesquisas René Rachou/
Fundação Oswaldo Cruz - FIOCRUZ
MINAS - Belo Horizonte (MG) - Brasil
2) Universidade do Estado de Minas Gerais
- UEMG - Belo Horizonte (MG) - Brasil
3) Universidade Federal de São João del-
Rei - UFSJ - São João del-Rei (MG) - Brasil
Recebido em: 10/07/2013 Revisado em: 07/01/2014 Aceito em: 08/05/2014 


\section{RESUMEN}

Objetivo: Investigar el conocimiento de los profesionales de la salud del municipio endémico del estado de Minas Gerais-Brasil sobre la Leishmaniasis. Métodos: Se realizó un estudio transversal con 228 profesionales (95 agentes de zoonosis, 83 agentes comunitarios de salud, 18 médicos, 17 enfermeros, 8 dentistas y 7 veterinarios) del municipio de Divinópolis - Minas Gerais entre julio y noviembre de 2009. Se utilizó un cuestionario estructurado, previamente validado con cuestiones objetivas sobre la enfermedad. Los análisis fueron realizados a través del programa Statistical Analysis System. Resultados: La categoría profesional que presentó mejor media de aciertos fue los veterinarios $(8,3)$, seguido de los médicos (8,1), mientras los agentes comunitarios de salud tuvieron la menor media $(6,7)$. Las respuestas adecuadas con menos porcentaje de aciertos fueron: medidas preventivas (42,5\%), manifestación clínica (25,9\%), nombres populares $(20,6 \%)$ y transmisión $(20,2 \%)$. A pesar de los agentes comunitarios de salud y zoonosis presentaren mayor porcentaje de respuestas incorrectas, en las demás cuestiones fueron ellos los que tuvieron más aciertos de las medidas preventivas, junto a los veterinarios. Conclusión: Se evidenció lagunas conceptuales en los profesionales de la salud de la investigación lo que refuerza la necesidad de implementación de procesos de educación permanente de los mismos, contextualizando las informaciones de Leishmaniasis y la realidad estudiada.

Descriptores: Leishmaniasis; Conocimiento; Recursos Humanos en Salud; Atención Primaria de Salud; Enfermedades Endémicas.

\section{INTRODUÇÃO}

As leishmanioses caracterizam-se por doenças infecciosas, não contagiosas, causadas por protozoários de diversas espécies do gênero Leishmania sp. A transmissão ocorre com a picada de pequenos insetos do gênero Lutzomyia que se infectam em reservatórios silvestres (raposa, marsupiais, primatas e roedores) e domésticos (cão). O ciclo biológico do Lutzomyia sp. se inicia com a deposição dos ovos em locais úmidos, ricos em matéria orgânica e de pouca luminosidade, não havendo fase evolutiva na água ${ }^{(1-3)}$.

Nas Américas, as leishmanioses apresentam duas principais categorias clínicas, associadas a uma gama de sintomas e graus diferentes de severidade: Leishmaniose Visceral (LV) e a Leishmaniose Tegumentar Americana (LTA). A LV apresentou expansão geográfica e urbanização nos últimos anos, gerando variação eco-epidemiológica de transmissão nas diversas regiões do Brasil, devido a fatores como evolução da ecologia/biologia dos vetores e do parasita, correlação entre infecção canina/humana, dentre outros $^{(4)}$. A LTA apresenta diferentes perfis epidemiológicos e padrões de transmissão em decorrência de modificações socioambientais ${ }^{(5,6)}$.
O caráter insidioso e a sintomatologia inespecífica das leishmanioses dificultam seu diagnóstico. Este fato, aliado ao desconhecimento dos profissionais de saúde, pode levar a atrasos graves na detecção da doença, o que, no caso da $\mathrm{LV}$, pode ser fatal ${ }^{(7)}$. A educação em saúde pode ser uma estratégia capaz de minimizar tais desfechos, pois permite conjugar importantes conceitos do ciclo e sintomatologia das zoonoses aos hábitos e às atitudes da população local. Com essa aproximação, aplicar ações preventivas/curativas realmente eficazes e duradouras torna-se mais tangível, pois elas são desenvolvidas com base nas percepções das comunidades $\operatorname{afetadas}^{(8)}$. Tal processo de educação em saúde deve ser contínuo, permitindo gerar vínculos de compromisso e corresponsabilidade entre a população e os que compõem o sistema de saúde ${ }^{(9)}$.

No Brasil, esse processo é protagonizado pelo Sistema Único de Saúde (SUS), pautando-se nos eixos da equidade, universalidade e integralidade. Dentro deste sistema, tem-se a atenção básica como o primeiro nível de atenção do SUS, o qual se constitui de equipes multiprofissionais - médicos, enfermeiros, dentistas e agentes comunitários de saúde que devem desenvolver a responsabilidade sanitária nas comunidades adscritas ao seu território, considerando suas características socioculturais ${ }^{(10)}$. A atuação conjunta desses profissionais pode facilitar a disseminação do conhecimento $\mathrm{e}$, progressivamente, efetivar o ciclo informativo e preventivo sobre enfermidades como as leishmanioses.

Entretanto, a literatura científica é escassa no que se refere a avaliar o conhecimento e as atitudes dos profissionais sobre as leishmanioses. Já foi demonstrado conhecimento pobre sobre a sintomatologia da doença, acompanhamento clínico inadequado na unidade de saúde com repercussão no sucesso do tratamento e ações impositivas em detrimento de uma abordagem dialógica de educação popular ${ }^{(8,11-12)}$.

O fato do programa nacional de controle das leishmanioses não ter sido capaz de conter o avanço da doença pelo país traz questionamentos sobre o papel desempenhado pelos profissionais da saúde no âmbito institucional e reforça a hipótese de que seu conhecimento, apesar de imprescindível, seja incipiente para contribuir com o sucesso deste.

No Brasil, entre os anos de 2007 e 2013, foram notificados 26.112 casos da LV e 159.301 casos de LTA $^{(13)}$. Em Divinópolis, Minas Gerais, ambas as formas da doença se encontram em expansão. Na década de 1990, o município registrou 135 casos da forma tegumentar e entre os anos 2007 e 2013, foram $54^{(13,14)}$. A LV é uma manifestação mais recente, tendo sido registrado 15 casos da doença no mesmo período ${ }^{(13)}$. Estudos no município demonstraram desconhecimento da população e dos professores acerca das leishmanioses, verificando-se fragmentação do saber ao considerar o contexto científico da enfermidade ${ }^{(15,16)}$. 
Também foi relatada no local a existência de flebotomíneos, vetores naturalmente infectados, em fragmentos de matas no perímetro urbano ${ }^{(14,17)}$ e alta prevalência de leishmaniose canina $^{(15)}$.

Diante do exposto, se percebe que a situação endêmica do município requer ações preventivas efetivas da equipe de saúde para conter o aumento do número de casos da doença em Divinópolis. Por isso, aventou-se a hipótese de que o conhecimento desses profissionais pode ser insatisfatório e pouco articulado com a população para lidar com a realidade da doença no município. Assim, o presente estudo teve como objetivo investigar o conhecimento sobre as leishmanioses dos profissionais de saúde de um município endêmico do estado de Minas Gerais-Brasil.

\section{MÉTODOS}

O presente estudo caracterizou-se por uma abordagem quantitativa, do tipo transversal, realizada em Divinópolis, área endêmica de Minas Gerais-Brasil, no período de julho a novembro de 2009.

Divinópolis está localizada na região centro-oeste do estado (20 8 '21"S e 44\%53'17" O) e tem população estimada de 213.016 habitantes $^{(18)}$. O município constitui-se em um pólo da região centro-oeste do estado, destacandose nos campos industrial e comercial - vestuário e siderurgia - o que exerce forte poder de atração sobre os municípios vizinhos ${ }^{(19)}$. Historicamente, a cidade foi uma região endêmica para a doença e apresentou um surto epidêmico de LTA, entre os anos de 1989 e 1990, em que o número de casos humanos passou de 29 para 79 por ano, respectivamente ${ }^{(15,20)}$. Posteriormente, surgiram os casos de LV nos anos de 2009 e 2010.

Participaram do estudo 228 profissionais de saúde, sendo 95 agentes de zoonoses (AZ), 83 agentes comunitários de saúde (ACS), 18 médicos, 17 enfermeiros e 8 dentistas em exercício nas unidades públicas de saúde do município e pelos 7 médicos veterinários atuantes em todas as clínicas veterinárias do município. Adotou-se como critério de inclusão estar presente na unidade ou clínica no dia agendado para a aplicação do questionário.

Para a formulação do instrumento de coleta, utilizaram-se conceitos relevantes sobre a doença, baseados em trabalhos semelhantes descritos na literatura ${ }^{(7,21-25)}$. Elaborou-se um questionário autoaplicável com 9 perguntas objetivas, compostas por 6 opções de resposta com somente uma alternativa correta.

A validação do questionário ocorreu mediante sua aplicação a 27 indivíduos com nível de escolaridade semelhante ao dos participantes deste estudo. A partir da detecção dos problemas no entendimento de algumas questões, efetuou-se a reformulação das mesmas.
Analisaram-se os resultados através da estatística descritiva e apresentados através de frequência absoluta e relativa, utilizando o programa Statistical Analysis System (SAS).

O presente trabalho foi aprovado no Comitê de Ética em Pesquisa da FUNEDI/UEMG (n 16/2009), em respeito à Resolução 196/96 do Conselho Nacional de Saúde, atualmente revogada pela 466/12 do Conselho Nacional de Saúde. Os profissionais receberam esclarecimentos sobre os objetivos, riscos e benefícios do presente estudo através do termo de consentimento livre e esclarecido.

Convidaram-se os profissionais de saúde do serviço público (médicos, enfermeiros, dentistas e ACS/AZ) a participar pela secretaria de saúde mediante carta de colaboração esclarecendo o teor da pesquisa. Contataram-se os médicos veterinários diretamente na clínica veterinária para agendamento. Entrevistaram-se os ACS e AZ durante um encontro no auditório da Fundação Educacional de Divinópolis e os demais profissionais, na própria unidade de saúde ou clínica veterinária a partir de agendamento prévio.

\section{RESULTADOS}

Dos 228 profissionais entrevistados, 65,6\% $(n=149)$ eram do sexo feminino, $50,7 \%(n=115)$ possuíam o ensino médio completo e $43,6 \%(n=99)$ apresentavam renda entre 1 e 3 salários mínimos (Tabela I).

Tabela I - Características socioeconômicas dos profissionais de saúde e veterinários de Divinópolis, Minas Gerais.

Características socioeconômicas Frequência Percentual

Sexo

Masculino 79

Feminino

149

65,6

\section{Escolaridade}

Primário Incompleto

Médio Incompleto

$1 \quad 0,4$

Médio Completo

18

7,9

Técnico

116

50,7

Superior Incompleto

3

1,3

Superior Completo

21

9,3

Remuneração

Entre 1 a 3 salários

30,4

Entre 4 a 5 salários

100

43,6

Mais de 5 salários
58

25,6

70 


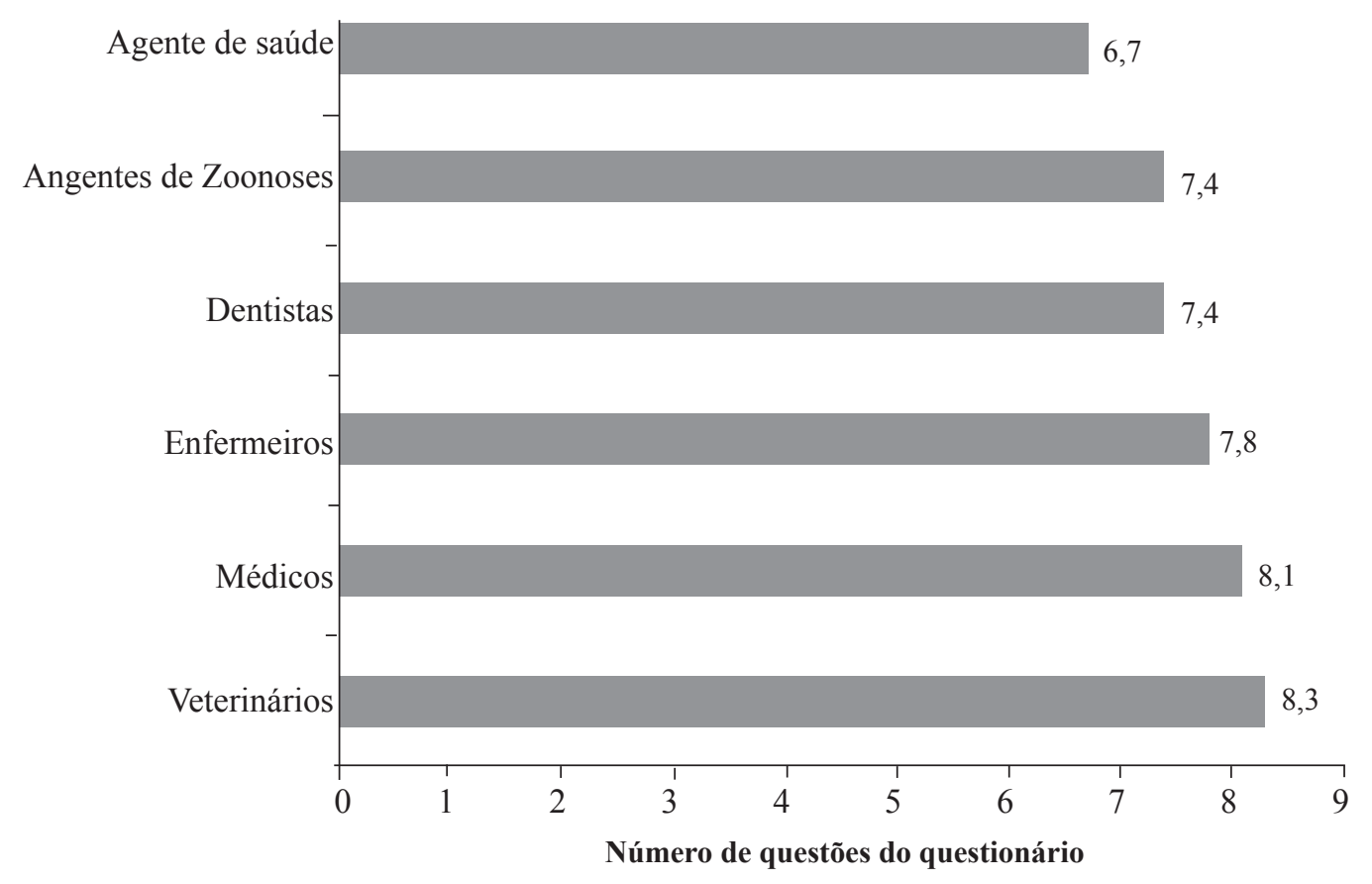

Figura 1 - Distribuição das médias de acerto sobre as leishmanioses dos diferentes profissionais de saúde entrevistados. Divinópolis-Minas Gerais, 2009.

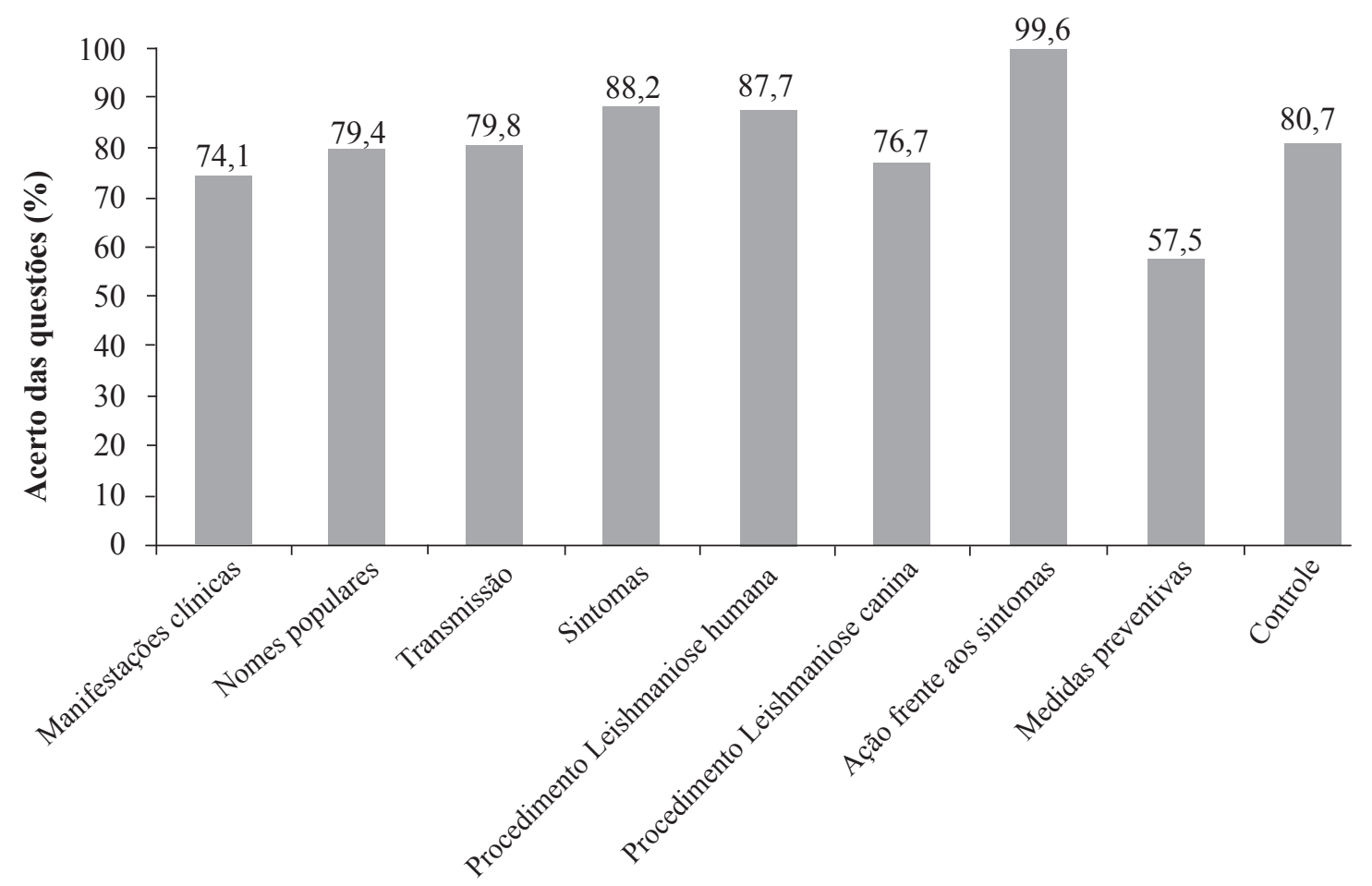

Figura 2 - Análise do desempenho geral dos profissionais de saúde entrevistados em cada questão sobre as leishmanioses. Divinópolis-Minas Gerais, 2009. 
Dentre as 9 questões apresentadas, os profissionais de saúde apresentaram média de acerto de 7,6 questões. Maior destaque para veterinários, com média de 8,3 , e para os ACS, menor média com 6,7 (Figura 1).

A Figura 2 mostra o desempenho dos entrevistados em cada questão. Todas as perguntas apresentaram índice de acerto semelhante, exceto quando foi abordado o assunto "medidas preventivas".

Ao analisar 4 questões com os piores resultados (medidas preventivas, manifestações clínicas, nomes populares da doença e transmissão) em relação os diferentes profissionais investigados, evidenciou-se discrepância entre os desempenhos destes profissionais, principalmente com relação às respostas dos ACS e AZ (Figura 3). Por outro lado, na questão relativa às medidas preventivas, esses profissionais, juntamente com os veterinários, detiveram maior conhecimento do que os demais atuantes na saúde pública, ainda que o percentual de acerto não tenha sido elevado.

Referente a medidas preventivas, este aspecto apresentou 57,5\% (n=131) de respostas adequadas ("manter a área externa da moradia limpa"). Este percentual foi influenciado pela escolha de alternativas equivocadas, como "cobrir as caixas d'água e não deixar águas empoçadas" apontada por $12,6 \%(\mathrm{n}=12)$ dos AZ, 21,7\% $(\mathrm{n}=18)$ dos ACS, $28,6 \%(\mathrm{n}=2)$ dos veterinários, $38,9 \%(\mathrm{n}=7)$ dos médicos,
$43,7 \%(n=7)$ dos enfermeiros e $50 \%(n=4)$ dos dentistas, indicando ações de prevenção à dengue.

No questionamento sobre as formas clínicas das leishmanioses, 74,1\% $(\mathrm{n}=169)$ dos profissionais responderam corretamente "visceral e tegumentar". Porém, $12,5 \%(\mathrm{n}=1)$ dos dentistas afirmaram formas "clássica e hemorrágica" e 20,5\% ( $\mathrm{n}=17)$ dos ACS optaram por "sintomática e assintomática".

Sobre o conhecimento dos nomes populares da LV e LTA, obteve-se $79,4 \%(n=181)$ de respostas corretas ("calazar e úlcera de bauru"). Entretanto, 13,3\% ( $n=11)$ dos ACS afirmaram ser a enfermidade conhecida como leptospirose e $13,7 \% \quad(n=13)$ dos $A Z$ não souberam responder.

Quando questionados sobre a forma de transmissão das leishmanioses, 79,8\% (n=182) dos profissionais responderam a alternativa correta. Entretanto, $11,1 \%(\mathrm{n}=2)$ dos médicos, $12,5 \%(\mathrm{n}=1)$ dos dentistas e 10,9\% $(\mathrm{n}=9)$ dos ACS optaram erroneamente pela alternativa "pela picada do mosquito Aedes aegypti infectado" e 12,5\% (n=1) dos dentistas, $8,4 \%$ dos ACS $(n=7)$ e AZ $(n=8)$ pela alternativa "através da mordida de cães infectados".

Questionados sobre as ações que devem ser tomadas frente à manifestação da doença em humanos, 87,7\% $(n=200)$ dos entrevistados responderam corretamente "tratamento imediato", mas $25 \%(\mathrm{n}=2)$ dos dentistas, $7,2 \%$

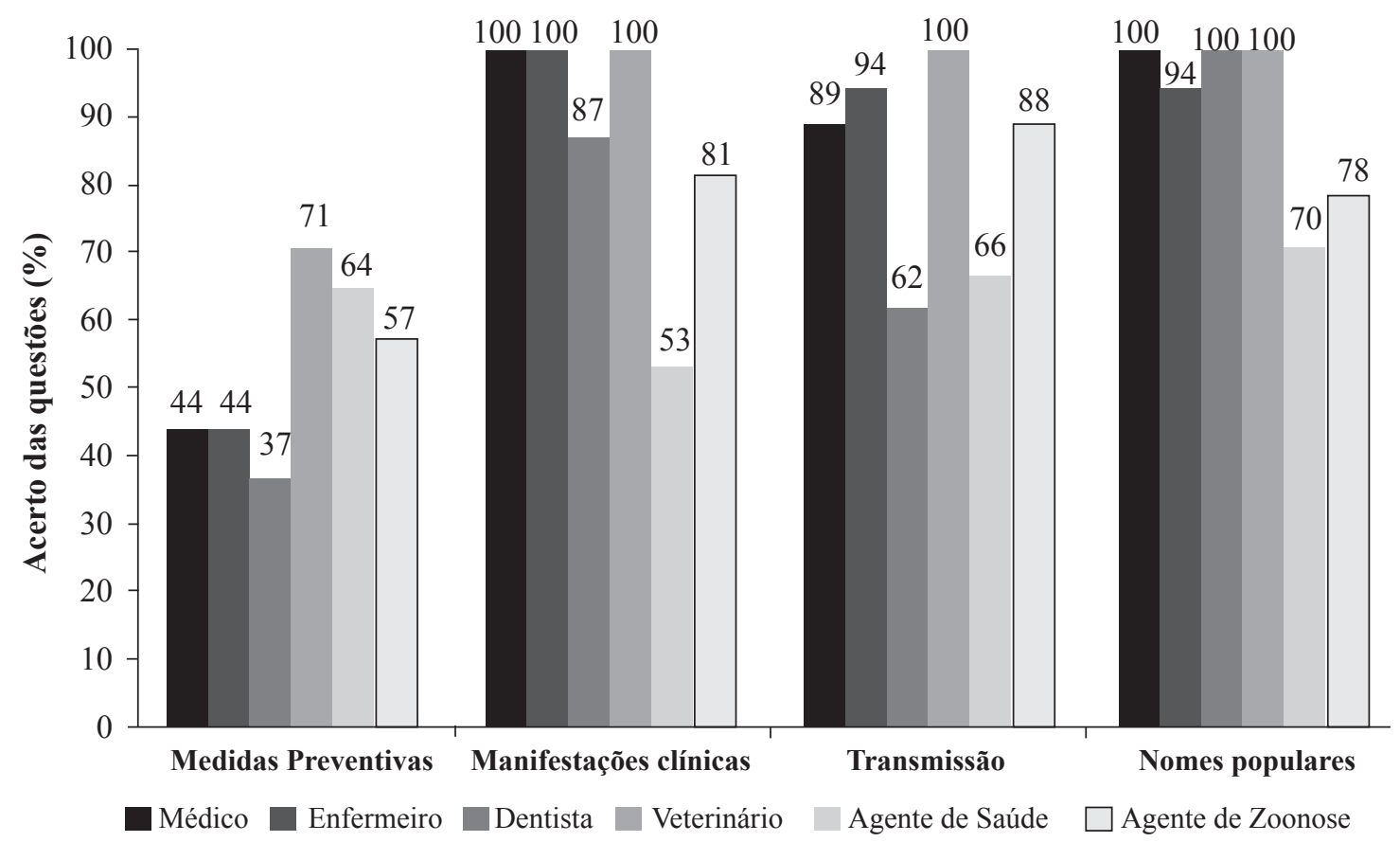

Figura 3 - Distribuição do percentual de acertos das questões sobre as leishmanioses com piores resultados pelos profissionais de saúde. Divinópolis-Minas Gerais, 2009. 
$(\mathrm{n}=6)$ dos ACS e 6,3\% (n=6) dos AZ assinalaram "vacinação de cães e seres humanos".

Observou-se que $80,7 \%(\mathrm{n}=184)$ dos profissionais de saúde escolheram adequadamente sobre o controle da enfermidade em questão ("eliminando cães infectados, eliminando flebotomíneos e tratando pacientes"). Porém, $28,6 \%(\mathrm{n}=2)$ dos veterinários, 20,5\% $(\mathrm{n}=17)$ dos ACS e $12,5 \%(n=1)$ dos dentistas e enfermeiros $(n=2)$ responderam que para o controle é preciso "eliminar água parada, vacinar os cães e tratar pacientes".

\section{DISCUSSÃO}

Demonstrou-se que os profissionais de saúde entrevistados apresentam diversas lacunas conceituais acerca das leishmanioses. $\mathrm{O}$ assunto de menor domínio foi com relação às medidas preventivas - percentual geral de acerto de $57,5 \%$. Isso pode refletir uma conduta focada, principalmente, no diagnóstico e tratamento dos pacientes ou animais infectados em detrimento de uma orientação preventiva fundamental para se evitar novos casos da doença ou sua reincidência.

A associação errônea entre ciclo biológico dos vetores da dengue e da leishmaniose evidencia essa discrepância, pois os vetores destas doenças possuem ciclos de vida distintos. Informação e educação adequadas são importantes na construção de cenários que favoreçam a prevenção em áreas endêmicas, sendo os profissionais, neste contexto, tanto disseminadores do conhecimento como parte integrante da população( ${ }^{(26)}$. Por isso suas percepções precisam ser aprimoradas a fim de permitir o controle da doença, objetivo que deve ser alcançado através de políticas públicas apropriadas que perpassem a atuação destes trabalhadores.

Os ACS e AZ são os atores mais próximos da população, sendo suas ações essenciais na divulgação de informações e detecção de casos suspeitos. Estudos sobre as percepções dos agentes quanto à dengue, por exemplo, demonstraram existir proximidade entre as representações dos agentes e o conhecimento científico, fato que pode surtir efeito positivo em suas práticas ${ }^{(27)}$, porém essa prática está pautada em prescrições e recomendações verticalizadas, desvalorizando o diálogo com a população ${ }^{(28)}$.

Outros estudos mostram existir confusão entre o nome da LV e o da leptospirose, não apenas pelos agentes de zoonoses, mas também por outros profissionais do Programa de Saúde da Família (PSF), como os enfermeiros, em pesquisa realizada na região metropolitana de Belo Horizonte $^{(7)}$ Esse fato também foi observado no presente estudo, em que $13,3 \%$ dos ACS apontaram a leptospirose como um nome popular da leishmaniose.
As respostas inadequadas referentes à transmissão das leishmanioses se relacionaram principalmente ao papel do vetor e do cão no ciclo da doença. A indicação do vetor Aedes aegypti como transmissor da enfermidade demonstra que, apesar de saberem que a leishmaniose é transmitida por um inseto, estes trabalhadores desconhecem o nome deste. Alguns profissionais afirmaram ainda que a transmissão poderia acontecer através da mordida de cães infectados $(12,5 \%$ dos dentistas e $8,4 \%$ dos ACS/AZ), associação igualmente verificada em um estudo realizado pela Universidade Federal do Paraná, em que 50\% dos ACS entrevistados afirmaram ocorrer a transmissão da leishmaniose pela mordida de cães ${ }^{(29)}$.

O cão é o principal reservatório da doença em ambiente urbano e tem, no sacrifício dos soropositivos, uma das principais medidas de controle adotadas atualmente. Duas vacinas para leishmaniose canina encontram-se disponíveis no mercado, porém a eficácia e a verificação de uma imunoproteção adequada estão em fases de testes ${ }^{(30)}$. Contudo, o Ministério da Saúde preconiza a eutanásia dos cães soropositivos e não reconhece a vacinação como uma medida eficaz no controle da enfermidade.

No presente estudo foi observado que $16,7 \%$ dos médicos afirmaram que o tratamento se dá apenas "na fase crônica da doença". Estudo sobre o histórico da epidemiologia da LTA e suas perspectivas de controle no Brasil demonstrou que o despreparo das unidades de saúde para o diagnóstico da doença consiste em um grande obstáculo para a abordagem precoce do doente ${ }^{(31)}$. Os mesmos autores também observaram a existência de um longo período entre a suspeita e o diagnóstico, ocasionado, em parte, pelas faltas de capacidade diagnóstica e de capacitação técnica dos profissionais de saúde.

O diagnóstico diferencial é de grande importância para evitar cicatrizações desfigurantes e mutilações, principalmente no caso de Leishmaniose Mucocutânea, que é pouco prevalente ${ }^{(32)}$. Dessa forma, os dentistas devem ser capacitados para o diagnóstico precoce de lesões bucofaríngeas, as quais podem ser podem ser causadas por uma variedade de doenças - paracoccidioidomicose, neoplasias, laringites, dentre outras ${ }^{(32,33)}$. O diagnóstico tardio resulta na elevação das taxas de letalidade das leishmanioses, principalmente da LV, tendo sido considerado um fator de risco para a morte, razão pela qual a capacitação dos médicos do PSF deve ser priorizada pela rede básica ${ }^{(34,35)}$.

Em uma nova perspectiva de promoção da saúde, com criação de laços de compromisso e corresponsabilidade entre profissionais e população, a educação em saúde desses trabalhadores pode garantir, juntamente com sua capacitação técnica, diagnósticos e tratamentos mais eficazes ${ }^{(4,9,31)}$. Dessa forma, um novo diálogo pode ser estabelecido com a população, tornando possível introduzir práticas coletivas 
de prevenção que evitem o adoecimento dos usuários, indo além do processo de cura.

Dessa forma, sugere-se que a equipe de saúde e os veterinários possam ser capacitados através de uma educação continuada contextualizada à realidade do município. Espera-se que os resultados compilados na presente pesquisa contribuam para melhorar a relação paciente/ profissional no contexto plural e dinâmico das leishmanioses, reorientando a prática no sentido da prevenção, promoção e reabilitação dos usuários em detrimento do foco puramente biomédico da doença.

\section{CONCLUSÃO}

Evidenciaram-se lacunas conceituais nos profissionais de saúde participantes da pesquisa, reforçando a necessidade de implementar processos de educação permanente destes profissionais, contextualizando as informações sobre as leishmanioses à realidade estudada.

\section{AGRADECIMENTOS}

Ao Centro de pesquisas René Rachou (CPqRR), à Fundação Educacional de Divinópolis (FUNEDI) e à Fundação de Amparo à Pesquisa de Minas Gerais (FAPEMIG - APQ-01657-11) pelo apoio financeiro. Ao Professor Msc. Fabrizio Furtado de Sousa e ao pesquisador Márcio Cleib Pereira pelo apoio na execução do projeto. À viabilização da pesquisa pelo então Secretário Adjunto de Saúde da Secretaria Municipal de Saúde de Divinópolis, Gilmar Santos.

\section{Conflitos de Interesse}

Todos os autores contribuíram efetivamente para a realização deste trabalho, se responsabilizando pelo seu conteúdo e não apresentam qualquer conflito de interesse com companhias/ instituições ou com assunto abordado.

\section{REFERÊNCIAS}

1. Talmi-Frank D, Kedem-Vaanunu N, King R, BarGal GK, Edery N, Jaffe CL, et al. Leishmania tropica infection in golden jackals and red foxes, Israel. Emerg Infect Dis. 2010;16(12):1973-5.

2. Esch KJ, Petersen CA. Transmission and epidemiology of zoonotic protozoal diseases of companion animals. Clin Microbiol Rev. 2013;26(1):58-85.

3. Lara-Silva FO, Barata RA, Michalsky EM, Ferreira EC, Lopes MOG, Pinheiro AC, Fortes-Dias CL, Dias ES. Rattus norvegicus (Rodentia: Muridae) Infected by
Leishmania (Leishmania) infantum (syn. Le. chagasi) in Brazil. Biomed Res Int. 2014;2014:1-7.

4. Gontijo CMF, Melo MN. Leishmaniose visceral no Brasil: quadro atual, desafios e perspectivas. Rev Bras Epidemiol. 2004;7(3):338-49.

5. Silva AF, Latorre MRDO, Galati EAB. Fatores relacionados à ocorrência de leishmaniose tegumentar no Vale do Ribeira. Rev Soc Bras Med Trop. 2010;43(1): 46-51.

6. Negrão GN, Ferreira MEMC. Considerações sobre a leishmaniose tegumentar americana e sua expansão no território brasileiro. Revista Percurso. 2014;6(1):14768.

7. Luz ZMP, Schall V, Rabello A. Evaluation of a pamphlet on visceral leishmaniasis as a tool for providing disease information to healthcare professionals and laypersons. Cad Saúde Pública. 2005;21(2):606-21.

8. Borges BKA, Silva JA, Haddad JPA, Moreira EC, Magalhães DF, Ribeiro LML, et al. Presença de animais associada ao risco de transmissão da leishmaniose visceral em humanos em Belo Horizonte, Minas Gerais. Arq Bras Med Vet Zootec. 2009;61(5):1035-43.

9. Oliveira SF, Albuquerque FJB. Family health program: an analysis from the beliefs of their service providers. Psicol Soc. 2008;20(2):237-46.

10. Ministério da Saúde (BR), Secretaria de Atenção à Saúde, Departamento de Atenção Básica. Vigilância em saúde: Dengue, Esquistossomose, Hanseníase, Malária, Tracoma e Tuberculose. Brasília: Ministério da Saúde; 2008.

11. Lima MVN, Oliveira RZ, Lima AP, Felix MLO, Silveira TGV, Rossi RM, et al. Atendimento de pacientes com leishmaniose tegumentar americana: avaliação nos serviços. Cad Saúde Pública. 2007;23(12):2938-48.

12. Silva SM. As ações de educação popular praticadas pelos agentes comunitários de saúde na abordagem das questões de saúde em Ceilândia-DF [monografia]. Brasília: Universidade de Brasília; 2013.

13. Sistema de Informação de Agravos de Notificação SINAN. Brasília: Leishmaniose: casos confirmados notificados no Sistema de Informação de Agravos de Notificação [acesso em 2014 Out ]. Disponível em: http://dtr2004.saude.gov.br/sinanweb/index. php?name=Tnet

14. Nascimento BWL, Saraiva L, Teixeira Neto RG, Meira PCLS, Sanguinette CC, Tonelli GB, et al. Study of sand flies (Diptera: Psychodidade) in visceral and cutaneous 
leishmaniasis area in central western of Minas Gerais state - Brazil. Acta Trop. 2013;125(3):262-8.

15. Margonari C, Menezes JA, Rocha MN, Maia KN, Oliveira ME, Fonseca AL, et al. Public Knowledge about and Detection of Canine Visceral Leishmaniasis in Urban Divinópolis, Brazil. J Trop Med. 2012;2012:114.

16. França VH, Margonari CS, Schall VT. Percepção de professores do ensino básico em relação as suas práticas educativas sobre leishmanioses: um estudo em área endêmica de Minas Gerais. Ensaio Pesquisa Educ Ciências. 2013;15(3):35-51.

17. Margonari C, Soares RP, Andrade-Filho JD, Xavier DC, Saraiva L, Fonseca AL, et al. Phlebotomine sand flies (Diptera: Psychodidae) and leishmania infection in Gafanhoto Park, Divinópolis, Brazil. J Med Entomol. 2010;47(6):1212-9.

18. Instituto Brasileiro de Geografia e Estatística IBGE. Brasília: Sinopse do censo demográfico 2010. Brasília; 2013 [acesso em 2013 Maio 30]. Disponível em: http://www.ibge.gov.br/cidadesat/xtras/temas. php? $\operatorname{codmun}=312230 \&$ idtema $=1$

19. Alvim AMM, Carvalho PFB, Oliveira PAB. Análise das dinâmicas econômica e populacional da Microrregião de Divinópolis. Cad Geografia. 2007;17(28):163-80.

20. Prefeitura Municipal de Divinópolis, Departamento de Epidemiologias. Registro de Leishmanioses. Divinópolis; 2009.

21. Borges BKA, Silva JA, Haddad JPA, Moreira EV, Magalhães DF, Ribeiro LML, et al. Avaliação do nível de conhecimento e de atitudes preventivas da população sobre a leishmaniose visceral em Belo Horizonte, Minas Gerais, Brasil. Cad Saúde Pública. 2008;24(4):777-84.

22. Silva-Nunes M, Cavasini CE, Silva NS, Galati EAB. Epidemiologia da Leishmaniose Tegumentar e descrição das populações de flebotomíneos no município de Acrelândia, Acre, Brasil. Rev Bras Epidemiol. 2008;11(2):241-51.

23. Ampuero J, Urdaneta M, Macêdo VO. Factores de riesgo para la transmisión de leishmaniases cutânea em niños de 0 a 5 años en un área endémica de Leishmania (Viannia) braziliensis. Cad Saúde Pública. 2005;21(1):161-70.

24. Aparicio C, Bitencourt MD. Modelagem espacial de zonas de risco da leishmaniose tegumentar americana. Rev Saúde Pública. 2004;38(4):511-6.
25. Sosa-Estani SS, Segura EL, Gomez A, Salomón OD, Peralta M, Coutada V, et al. Leishmaniose cutânea no Norte da Argentina: fatores de risco identificados num estudo caso-coorte em três municípios de Salta. Rev Soc Bras Med Trop. 2001;34(6):511-7.

26. Mondal D, Alama MS, Karim Z, Haquea R, Boelaert $\mathrm{M}$, Kroeger A. Present situation of vector-control management in Bangladesh: A wake up call. Health Policy. 2008;87(3):369-76.

27. Corrêa LMC. As representações sociais dos agentes de controle de zoonoses sobre a dengue em Uberlândia, MG [dissertação]. Uberlândia: Universidade Federal de Uberlândia; 2012.

28. Oliveira GLA. Prevenção e controle da dengue no município de Sabará/MG: análise de materiais educativos impressos e das representações sociais de agentes de controle de endemias [dissertação]. Belo Horizonte: Centro de Pesquisa René Rachou; 2012.

29. Almeida JC, Grazziotin AL, Marinho AP, Bollmann C, Amaral CH, Skalski J, et al. Percepção sobre zoonoses de professores e agentes comunitários de saúde no município de Quatro Barras, Paraná. In: Anais do IV Congresso latino americano/ X Congresso Brasileiro de Higienistas de Alimentos, III Encontro de Centros de Controle de Zoonoses e II Encontro do Sistema Brasileiro de Inspeção de Produtos de Origem Animal, Florianópolis, 2009. São Paulo: DPI Estudio e Editore LTDA; 2009. v. 23. p. 602-3.

30. Campino L, Maia C. Epidemiologia das leishmanioses em Portugal. Acta Med Port. 2010;23(5):859-64.

31. Basano AS, Camargo LMA. Leishmaniose tegumentar americana: histórico, epidemiologia e perspectivas de controle. Rev Bras Epidemiol. 2004;7(3):328-37.

32. Mota LAA, Miranda RR. Manifestações dermatológicas e otorrinolaringológicas na Leishmaniose. Arquivos Int Otorrinolaringol. 2011;15(3):376-81.

33. Moraes BT, Amorim Filho FS, Caporrino Neto J, Saraceni Neto P, Melo Júnior JES. Leishmaniose Laríngea. Int. Arch. Otorhinolaryngol. 2012;16(4):5236.

34. Ministério da Saúde (BR), Secretaria de Vigilância em Saúde, Departamento de Vigilância Epidemiológica. Manual de vigilância e controle da leishmaniose visceral. Brasília: Ministério da Saúde; 2006.

35. Collin S, Davidson R, Ritmeijer K, Keus K, Melaku Y, Kipngetich S, et al. Conflict and kala-azar: determinants of adverse outcomes of kala-azar among patients in southern Sudan. Clin Infect Dis. 2004;38(5):612-9. 


\section{Endereço do primeiro autor}

Júlia Alves Menezes

Programa de Pós-Graduação em Saúde Coletiva do Centro de Pesquisas René Rachou/ Fundação Oswaldo Cruz Laboratório de Educação em Saúde e Ambiente Avenida Augusto de Lima, 1715

Bairro: Barro Preto

CEP: 30.190-002 - Belo Horizonte - MG - Brasil

E-mail: julia.menezes@cpqrr.fiocruz.br

\section{Endereço de correspondência}

Carina Margonari de Souza

Laboratório de Educação em Saúde e Ambiente

Avenida Augusto de Lima, 1715

Bairro: Barro Preto

CEP: 30.190-002 - Belo Horizonte - MG - Brasil

E-mail: margonari@cpqrr.fiocruz.br 